\title{
The 20th Century
}

L. E. J. (Bertus) Brouwer (1882-1966) was a geometer and topologist and the founder of intuitionism. He spent most of his life at the University of Amsterdam, and is remembered for his invariance and fixed-point theorems in topology and for his refusal to accept proofs by contradiction.

Wacław Sierpiński (1882-1969) was a Polish mathematician who made outstanding contributions to set theory, point-set topology, and number theory. The fractal pattern known as the Sierpiński triangle (also called the Sierpinski gasket) was described by him in 1915.

Eduard Čech (1893-1960) was a Czech mathematician who worked in topology and differential geometry. He is remembered for Čech homology and the Stone-Čech compactification of topological spaces.

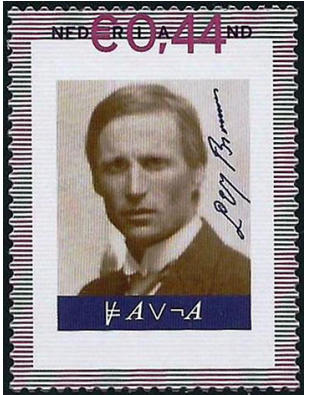

L. E. J. (Bertus) Brouwer

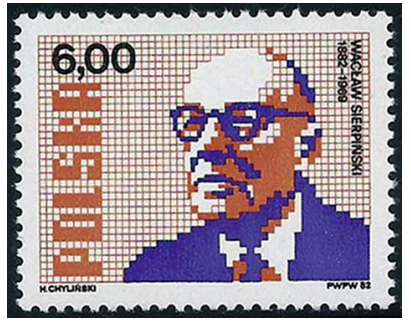

Wacław Sierpiński

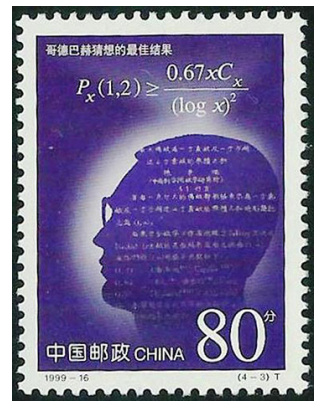

Chen Jing-Run

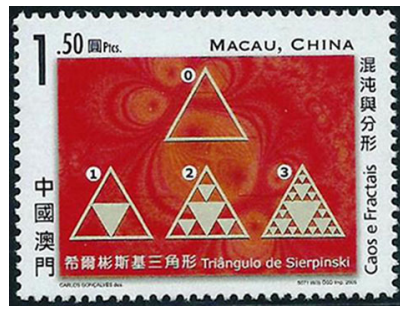

Sierpiński triangle claiming that every even integer greater than 2 can be written as the sum of two prime numbers (for example, $18=13+5)$. Although this still remains unproved in general, a partial result due to Chen Jingrun (1966) implies that every sufficiently large even integer can be written as the sum of a prime and an "almost-prime" (a number with at most two prime factors).

Fermat's "last theorem" asserts that for every integer $n>2$, there do not exist nonzero integers $x, y$, and $z$ for which $x^{n}+y^{n}=z^{n}$. Fermat proved this for $n=4$ with his "method of infinite descent," but it is unlikely that he had a general argument, despite his claims to have found one. In 1995, after a long and difficult struggle, it was eventually proved by Andrew Wiles; his name appears across the equal sign on the stamp issued by the Czech Republic in 2000.

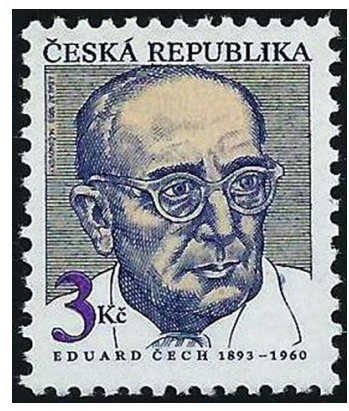

Eduard Čech

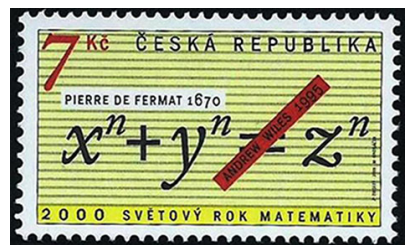

Andrew Wiles
Publisher's Note Springer Nature remains neutral with regard to jurisdictional claims in published maps and institutional affiliations. 